\begin{tabular}{|l|l|l|l|l|}
\hline Cuadernos I. Geográfica & 25 & pp. 125-137 & Logroño & 1999 \\
\hline
\end{tabular}

\title{
DESARROLLO SOSTENIBLE, TURISMO RURAL Y PARQUES CULTURALES
}

\section{BIELZA ${ }^{1}$}

RESUMEN: Se exponen los recientes cambios experimentados en el medio rural desde el productivismo tradicional a la terciarización. Se discuten las posibilidades turisticas del medio rural, y especialmente de la montaña media mediterránea, en el marco del desarrollo sostenible. Se explican las funciones y caracteristicas de los parques culturales.

ABSTRACT: In this paper the author shows the changes observed in the rural areas from the traditional agriculture until the pluriactive agriculture. The touristic possibilities of the rural areas, and specially of the Mediterranean mountain, are argued according to the theory of the sustainable development. Finally, the author explains functions and characteristics of the cultural parks.

Palabras clave: desarrollo sostenible, ecoturismo, turismo rural, parques culturales.

Key words: sustainable development, ecotourism, rural tourism, cultural parks.

Los cambios del medio rural europeo a lo largo del último siglo, primero desde el productivismo tradicional al tecnológico, después con el paso a una sociedad postindustrial y del ocio (mientras evolucionaba la política agraria comunitaria), han conducido a una pluriactividad y terciarización de los espacios rurales.

Entre las nuevas funciones del medio rural y especialmente de la montaña media mediterránea, poco impactada por el productivismo tecnológico, está el

1. Departamento de Geografía y Ordenación del Territorio. Universidad de Zaragoza. Ciudad Universitaria, s/n 50009 - ZARAGOZA. 
turismo rural que debe plantearse como una solución de desarrollo sostenible. Un planteamiento de estas características es el que se está haciendo en Aragón desde la figura del parque cultural, objeto de una ley (3-XII-97) que regula una realidad que protege y promociona unos territorios que contienen elementos relevantes de patrimonio cultural en un marco físico de valor paisajístico de cara a un desarrollo sostenible, a través del turismo rural.

\section{Los cambios del medio rural}

Entre los cambios del medio rural, habría que plantear el que se produce desde el productivismo tradicional al tecnológico, que aporta unas soluciones, pero indudablemente genera también unos problemas, especialmente desde el punto de vista social y ecológico. La política agraria comunitaria (P.A.C.), que se inicia en el 57, a partir de la configuración del Mercado Común y donde España entra tardíamente, va provocando nuevos problemas, que de alguna manera van a tener que dar lugar a soluciones, como la del turismo rural, en la Europa Comunitaria. $\mathrm{Al}$ incorporarse después España acumula sus propias disfunciones, a las aportadas por la P.A.C., no tanto inicial, como por la reforma de la misma. Todo ello nos va a llevar a la idea de que en el medio rural se va a ir pasando, unas veces de forma evolutiva y en otras ocasiones de forma más revolucionaria, desde un productivismo tradicional, enclavado claramente en sectores primarios, a un productivismo tecnológicamente intensivo, agresivo de alguna manera con el medio ambiente, como ha sucedido con la actividad industrial, para luego, a partir de una serie de incidencias de la reforma de la P.A.C. y de otra serie de cuestiones, como puedan ser las propias condiciones de la Ronda Urugay, acabar en un enfoque, -que empieza en las montañas- de buena parte del antiguo espacio rural como un espacio de ocio, un espacio terciarizado, un espacio complementario del espacio urbano para las poblaciones "estressadas" por la actividad urbana, por la actividad industrial o por las actividades terciarias avanzadas e intensivas.

\subsection{El cambio desde el productivismo tradicional al tecnológico}

Para situarnos en el comienzo de los cambios experimentados por el agro tradicional tendríamos que remontarnos aproximadamente a finales del siglo XIX, aunque en cada región española, la revolución tecnológica se produce en unas fechas algo diferentes. Pero evidentemente en las zonas transformadas hubo una mecanización-motorización, una introducción de la selección de semillas, una utilización del abonado químico, unas rotaciones más científicas que las tradicionales, el uso cada vez más masivo de insecticidas, fungicidas y productos químicos en general, provenientes de la industria.

Todos estos elementos tecnológicos generaron unas consecuencias de carácter económico, social y ecológico que se fueron sucediendo con mayor o menor 
agresividad, según los casos, a lo largo del siglo XX. En primer lugar, desde el punto de vista económico: el productivismo tecnológico deriva en un incremento de la productividad por persona ocupada, lo cual significa, a su vez, el éxodo rural, al irse eliminando puestos de trabajo. Ahora estamos viviendo una fase de pérdida de población laboral en la industria pero evidentemente de donde primero se eliminó y de una manera, al final verdaderamente dramática, es en el medio rural. No sólo se perdió población y de una forma masiva, quedando depauperadas las comunidades rurales, sino que también se perdió entramado social, o mejor comunitario (porque al fin y al cabo las sociedades rurales han funcionado en la época tradicional más como unas comunidades rurales). Lo grave de todo es, al final, la pérdida de masa social, como diría Unamuno al principio de este siglo, al hablar de las comuniades rurales. La pérdida de masa social, la pérdida del entramado social, la pérdida de iniciativas.

Pero no sólo se produce un incremento de productividad con estas consecuencias sociales negativas sino que hay, además, unos incrementos en los rendimientos por hectárea, una dependencia de la tecnología exterior, una dependencia de mercados exteriores, cada vez mayor, del mundo rural, con lo cual los precios se imponen desde fuera, lo que trae como consecuencia el distanciamiento entre las rentas del campo y las rentas de la ciudad. De una manera u otra es la ciudad con sus actividades industriales, con sủs actividades terciarias, la que rige las actividades del espacio rural. Y al final se lleva la mejor parte el que reparte, el que dicta los precios, que se ubica en las ciudades de los países desarrollados.

Paradójicamente, mientras se va produciendo este proceso, que queda bajo el epígrafe del cambio desde el productivismo tradicional al tecnológico, mientras se va hacia el distanciamiento entre los niveles de renta, desde el punto de vista cultural se va forjando un acercamiento de la cultura urbana al medio rural. Esto se genera fundamentalmente por los medios de comunicación y de modo especial por la televisión. Los cambios, a veces, son mucho más rápidos en el campo cuando se quieren imponer ideas o mensajes desde la televisión. Tienen mucho más impacto en el medio rural donde no hay una protección cultural y de valores urbanos ante los nuevos mensajes.

Desde el punto de vista ambiental habría que señalar que a partir de esa tecnologización de la agricultura, de esa introducción de técnicas cada vez más agresivas con maquinarias más potentes, con abonos químicos más sofisticados las agresiones al suelo rural han sido más intensas que anteriormente. Así por ejemplo tras las labores más profundas, cuando se abandonan agrícolamente los suelos en pendiente, estos experimentan una erosión posterior mucho más rápida que antes. Habría que señalar también la utilización de abonos químicos, de insecticidas, de fungicidas, su incidencia en la contaminación de suelos, en la 
degradación de acuíferos, en la contaminación de las aguas superficiales, hechos que no sucedian evidentemente en una agricultura tradicional, en que el ciclo natural del agua permitía recuperarse de los desechos orgánicos que se producían en el medio rural.

\section{2.- La politica comunitaria (P.A.C.)}

La P.A.C. en principio se centraba en la subvención a los precios con lo que se fue creando una agricultura hiperproductiva de costes elevados que tenía como objetivo primero el autoabastecimiento de alimentos para la Europa Comunitaria. Dicho objetivo se cumplió hasta la saciedad, hasta provocar la acumulación de alimentos en almacenes con difícil destino. El segundo objetivo que se marcó la política agraria comunitaria no se alcanzó; se trataba de aminorar las diferencias entre las rentas campo-ciudad y hoy en el medio rural europeo sigue habiendo más pobres que en el medio urbano (bien es verdad que la comparación no es tan fácil como a primera vista parece porque en el medio rural siempre hay un autoabastecimiento de determinados productos que no se produce en el medio urbano). Así por ejemplo: España tiene un 12\% de pobres en la ciudad y un 33\% de pobres en el medio rural. El Italia o en Francia, aunque las distancias no son tan fuertes, son todavía grandes y si no lo son mayores es porque en países como Francia hay una subvención todavía importante y además se trata de un medio rural más rico y productivo. Por otro lado la política agraria comunitaria ha favorecido, a determinados países, como Alemania, o Francia y evidentemente el distanciamiento es menor que en España.

La política agraria comunitaria desembocó en resultados distintos en la zona atlántica y en la zona mediterránea, de modo que no sólo se fueron provocando mayores disférencias campo-ciudad, sino que también se generaron distanciamientos entre la agricultura oceánico-atlántica de la Europa Comunitaria y la agricultura mediterránea. En realidad la P.A.C. va a favorecer sobre todo a la agricultura atlántica, hasta llegar a casos como el de los excedentes de los productos lácteos; en cambio la agricultura mediterránea tropieza con graves problemas estructurales, empezando por los propios problemas naturales que conocen bien nuestros campesinos. Aquí tenemos una abundancia de suelos en pendiente, frente a la plenitud de las grandes extensiones sobre las que se desarrolla la agricultura atlántica, un predominio de suelos excesivamente calcáreos, un déficit hídrico, que define la agricultura mediterránea y que sólo puede paliarse cuando existen unos regadíos suficientes. Por otro lado en países como en Grecia, Italia o España, nos encontramos con un tamaño medio de las explotaciones en la zona mediterránea muy bajo. Hay problemas, todavía, de minifundismo y de dispersión. Pero hay además problemas sociales o sociolaborales como el del envejecimiento de los activos. En España más de la mitad de los activos rurales tiene una edad supe- 
rior a los 55 años y por otro lado a pesar de que el medio mediterráneo ha expulsado muchos excedentes rurales, todavía queda un superávit que tiene que desaparecer en los próximos años en todas las penínsulas mediterráneas (aún teniendo en cuenta que la mitad de la población activa está trabajando a tiempo parcial).

Los problemas ambientales que se han provocado a partir de la política de la P.A.C. continúan los procesos señalados antes, propios de una intensificación agraria en determinadas zonas (contaminación atmosférica, hídrica y edáfica, intensificación de la erosión) a los que hay que añadir la homogeneización de paisajes frente a la diversidad tradicional. Esto último va a ser un inconveniente de cara a la utilización de determinados espacios para el turismo rural. Va a pasar como en las ciudades; aquellos espacios urbanos que se industrializaron y homogeneizaron, evidentemente son muy poco agradables para su recuperación, para su uso recreativo o terciario y, en cambio, aquellos que estuvieron fuera de este carácter uniformizador pueden recuperarse mejor. En el mundo rural sucede lo mismo.

Habría que decir también que el abandono de tierras, especialmente en la montaña mediterránea, está provocando problemas múltiples ambientales; que no porque se vaya la población hay una recuperación automática dé la naturaleza. Hay a veces problemas de erosión en las zonas antes cultivadas, hay en otros casos una recuperación vegetal de suelos, pero de forma desordenada, de modo que por ejemplo, se embastecen los pastos de cara a la ganadería. Pero sobre todo hay una invasión de la maleza. Los que viven en el mundo rural saben que ahora es más fácil un incendio que antes, cuando el sotobosque se utilizaba para combustible y había una limpieza periódica de la hojarasca y leña. Eso no lo limpia nadie ahora y por tanto hay mayores facilidades para los incendios, máxime cuando estos espacios son ocupados temporalmente por un turismo desordenado o unas visitas de viajeros de fin de semana. Por otra parte al abandonar los campesinos determinadas zonas, se pierden los principales guardianes del patrimonio natural y del patrimonio etnológico y cultural. Así, por ejemplo, se van abandonando viviendas rurales tradicionales de gran interés etnológico.

¿Qué pasa a partir de la reforma de la P.A.C., una vez que se conocen las citadas secuelas económicas, sociales y ambientales? ¿Qué pasa a partir del año 92? La reforma del 92 fue un reconocimiento de que existian todos estos problemas enunciados, de que se habían provocado secuelas negativas de orden social y ecológico. En aquellos momentos de principios de los 90 se estaba llevando a cabo una serie de reuniones mundiales que culminan en la Conferencia de Río en pro del desarrollo sostenible, había también reuniones en Euröpa de los ministros de Medioambiente y de Ordenación del Territorio en la misma línea. Pó tanto hay una preocupación creciente por los problemas ambientales. Paralelamente se com- 
prueba que hay una superproducción de alimentos en el seno de la Europa cormunitaria y que se está realizando una financiación auténticamente absurda, no consiguiendo elevar el valor de las rentas agrarias.

Por otro lado están las presiones de Estados Unidos, están las presiones que se llevan desde el GATT y este organismo al final con la ronda Uruguay acaba en una línea de liberalización de precios. El que los campesinos europeos se encuentren ante un mercado más abierto que antes, con menos barreras arancelarias, es evidente que conduce a una situación distinta.

Las soluciones de esa reforma de la P.A.C. son bien conocidas: subvención directa a los agricultores para paliar la liberalización de los precios, de manera que ahora mismo en España una cuarta parte de las rentas de las explotaciones agrarias provienen de esta vía subvencionadora, pero esto es pan para hoy y hambre para mañana, porque esto no se va a mantener eternamente. Hay una política de jubilaciones anticipadas que pretende que accedan jóvenes agricultores con una mentalidad más moderna, con más imaginación, que tiene que luchar por el futuro y eso evidentemente hace que las explotaciones sean más rentables que las de agricultores mayores a los que ya se les han ido sus hijos, que están mejor colocados en la ciudad y que evidentemente no van a hacer esfuerzos complementarios en la explotación de aquellos suelos marginales y más difíciles.

\section{Las funciones actuales del medio rural}

\subsection{De productivismo a la pluriactividad y la terciarización}

A partir de la situación anterior es cuando se plantean las funciones nuevas que tiene que desempeñar el medio rural: pasar de un productivismo muy centrado en el sector primario -de forma exclusiva-, como sucedía en el medio rural tradicional y también en el que se mecanizó, a una pluriactividad y a un uso terciario del espacio rural, en buena medida.

La pluriactividad en el medio rural comienza fundamentalmente en la montaña, que es donde antes se pierden las rentas, donde antes se pierde la población, donde antes aparece un turismo interesante y para donde los países más avanzados van elaborando una normativa adecuada para proteger estos espacios frágiles. Así por ejemplo la Ley de Montaña del país galo, en la que se proponen normas que facilitan la pluriactividad, que permiten al agricultor hacer otras actividades no agrícolas no gravándole impositivamente por todas ellas, si no sólo por una. Dentro de este marco de pluriactividad es donde hay que insertar la actividad del Turismo Rural. Pero habría que hacer también, antes de entrar en el turismo rural, otra serie de consideraciones. En primer lugar que esta plurifuncionalidad permite una mayor participación de la mujer en el mercado laboral. Es evidente que en la agricultura tradicional la mujer trabaja pero normalmente no 
aparecía ella como activa en las estadísticas del sector primario en España, donde no había manera de descubrir la participación de la mujer, porque normalmente no aparecía contabilizada. A partir de ahora tiene que aparecer por fuerza porque en la explotación familiar la mujer desempeña unas funciones económicas explícitas, especialmente en el turismo rural.

\subsection{El agricultor, agente de protección ambiental}

Pero el agricultor, además, empieza a contemplarse como un agente de protección ambiental. Es un descubrimiento reciente de los políticos comunitarios: el que mejor ha defendido y puede defender el medio ambiente es el agricultor tradicional. En esta línea política de fomentar la protección ambiental se concedieron subvenciones para dejar de cultivar un 15\% de las explotaciones que superarán las 20 hectáreas, con objeto de reforestar, de recuperar arbolado, eliminando los cultivos anuales. En esta tendencia se sitúa también el fomento del barbecho. Algo que les animaban a los agricultores, hasta hace 20 ó 30 años, a eliminar mediante rotaciones científicas ahora se trata de recuperar, con objeto de seguir una línea de agricultura ecológica poco productivista.

En definitiva esta línea ambientalista conduce a un desarrollo sostenible, dentro del espíritu de la conferencia de Río del 92, que se mencionaba antes; a considerar el medio rural como un espacio donde los recursos tienen que ser renovables. La sostenibilidad como concepto implica el que podamos dejar el patrimonio actual a las generaciones venideras y eso sólo se puede hacer si llevamos a cabo una actividad respetuosa con el medioambiente. Pero claro, entre tanto se producen contradicciones dentro de la Europa comunitaria, como puede ser el mandato del arranque de viñedos en más de 300.000 hectáreas que no oculta más que la presión de los remolacheros de azúcar procedentes de la Europa Atlántica. Una vez más quieren mantener una fácil salida de sus productos a costa del Sur, del vino de los países mediterráneos. Es evidente, que el azúcar de la remolacha asegura mejor el grado del vino pero la calidad del producto no es la misma. Pero eso son contradicciones, porque el arrancar cepas, sobre todo en los suelos en pendiente y pedregosos, significa facilitar la erosión. Aparte del problema social que conlleva se provoca un problema ambiental. Las contradicciones ambientales de la política comunitaria, cuentan en este caso con un buen ejemplo.

\subsection{Las funciones del medio rural mediterráneo}

El medio rural mediterráneo nos interesa porque es el medio en el que vivimos y el medio en el que se puede desarrollar en mayor medida un turismo rural. Nosotros en España exclusivamente tenemos, una franja norte, estrecha, de clima y de agricultura oceánica, el resto es mediterráneo, aunque en el interior haya que hablar de una continentalización del mismo. 
¿Cómo quedan ahora, los usos del suelo en la agricultura mediterránea? E1 balance es bastante sencillo y es que hay muy pocos suelos en la agricultura mediterránea -no sólo en España sino también en Italia, en Grecia, en el Sur de Francia- hay muy pocos suelos que por su nivel de modernización puedan competir con éxito con la agricultura del Norte en las mismas producciones. A estos pocos hay que añadir los enclaves de regadío de productos hortofrutícolas que, evidentemente no pueden darse en la Europa Atlántica y en los que llevamos ventaja, pero quedan reducidos a extensiones limitadas. En cambio, hay grandes espacios cultivados bajo las pautas de la política agraria comunitaria anterior que no son competitivos de ninguna manera y que lo van a ser mucho menos en un mercado más libre y más abierto al resto del mundo. $\mathrm{Y}$ esos suelos van a tener que sufrir un proceso similar al que sufrieron los suelos industriales, que se denominaron, "barbechos industriales". En el mundo rural mediterráneo a pesar de que estamos ya con muy poca gente en el campo aún hay que perder más en este tipo de agricultura. Hay que llegar a una reconversión, similar a los de aquellos "barbechos industriales" que se reconvirtieron en suelos para otros usos. Así en las zonas de la Lorena, que antes eran ocupadas por industrias sucias, hay ahora un magnífico paisaje verde, o bien unas industrias de alta tecnología con muy pocos obreros y con muy poco consumo de suelo, con lo cual el resto hay que adornarlo, hay que arreglarlo porque ya no son industrias contaminantes. ¿Pues bien qué hacemos con esos suelos agrícolas obsoletos? Esos suelos, esos barbechos ya no van a poder ser ni siquiera barbechos agrícolas, entonces habría que reforestar en la medida de lo posible, habría que dedicarlos a usos cinegéticos y también, por desgracia, tienen la vocación de cementerios de residuos, ya que son zonas alejadas que se han quedado despobladas y sin defensores territoriales. Pero si estamos hablando de suelos calcáreos, por ejemplo, habrá que tener mucho cuidado porque son suelos permeables y tenemos verdaderos problemas en sus acuíferos, mayores que con la agricultura tecnológica que soportaban anteriormente.

Nos quedan finalmente unos paisajes agrarios tradicionales que no se modernizaron, esos de montaña media, muchas veces, con bancales, con explotaciones en torno a casas de labranza dispersas, casas rurales tradicionales, que tienen un encanto paisajístico, y son capaces de atraer turismo rural. Les sucede lo mismo que a las pequeñas ciudades que no se industrializaron, que ahora cuentan en la nueva era de la competitividad entre ciudades, con una ventaja competitiva respecto de aquellas pequeñas ciudades que en un determinado momento optaron por la industrialización. Bueno pues en el mundo rural sucede igual: aquellos que mantuvieron el paisaje tradicional van a ser los que tengan una ventaja mayor. De la misma forma que aquellos cascos históricos con calles laberínticas, monumentos artísticos, casas antiguas y demás, tienen una mayor capacidad de atraer turis- 
DESARROLIO SOSTENIBLE, TURISMÓ RURAL Y PARQUES CULTURALES

mo que aquellas otras, que tiraron las construcciones antiguas e hicieron edificios funcionales que no atraen turismo de ninguna manera.

\section{El turismo rural como desarrollo sostenible del espacio rural}

\subsection{El turismo rural como función actual del medio rural.}

Más allá de la diferencia entre el turismo rural y el más restringido concepto de agroturismo o la inmersión de las dos ideas en la acepción del turismo verde o de turismo ecológico, o de la consideración del turismo de montaña e interior como contrapunto al turismo de playa, parece más importante situar el fenómeno históricamente: en Europa, el turismo rural se empieza a dar en los Altos Alpes hacia los años 50, antes que en nuestro Alto Pirineo. Antes, por la sencilla razón de que antes se abandona el medio rural en aquella montaña y de que en sus países limítrofes hay una demanda mayor, unas mejores posibilidades de que los urbanos, entre comillas, con medios de locomoción propios, etc. puedan interesarse por el medio rural.

Pero la explosión del turismo rural no se produce en Francia y en los países alpinos hasta el $2^{\circ}$ quinquenio de los 70 , coincidiendo con varios hechos. Una crisis económica fuerte, un hastío de la playa, sobre todo de la gente culturalmente más avanzada, con la consiguiente búsqueda de nuevos espacios recreativos. Todo ello conduce a que con una oferta más desarrollada en el mundo rural, con unas mejores viviendas, aunque antiguas, rehabilitadas, y con un traslado de la cultura urbana ya importante en Europa al mundo rural, pues se produzca una inclinación importante hacia el turismo rural. Y eso sucede no sólo en Francia, en Alemania o en Italia, ocurre también en España, aunque más lentamente.

Empieza a darse un abandono de tierras por la política agraria comunitaria, cuando se propicia el valor ecológico del paisaje agrario y se va preparando la legislación de los países más desarrollados desde el punto de vista ambiental. Una legislación adecuada, como la francesa de los parques regionales, que concilia protección con desarrollo rural sostenible.

Es evidente que con estos factores favorables se va desarrollando con más fuerza todo lo que es el turismo rural, que como duración media suele tener de 3 a 4 días. Es un turismo que se desarrolla evidentemente dentro del medio rural en las zonas de paisajes y de hábitats más atractivos. Es un turismo que encuentra un tipo de explotaciones muy diferenciadas, desde explotaciones modestas hasta explotaciones muy sofisticadas, en las que el turismo rural ya empieza a competir, incluso con ventaja, respecto de los establecimientos turísticos tradicionales. Eso es lo que en este momento ya empieza a darse en España de una manera incipiente en que el turismo convencional empieza a responder y a protestar porque comienzan a notar una competencia por parte del turismo rural. 
Este turismo ha de tener ayudas por parte de la Administración y ha de tener evidentemente un control. Ha de tener ayudas porque el turismo rural desempeña una misión distinta a la del turismo convencional. La Administración local, regional, nacional, e incluso europea, está propiciando ayudas a este sector con el objetivo, dentro de esa línea de pluriactividad, de mantener la población en el medio rural.

La Administración, ¿qué puede hacer? Puede hacer la publicidad de las casas rurales, puede hacer los catálogos turísticos, puede exigir calidad dando una normativa de calidad. Pero en nuestro país, la verdad es que la Administración Central no ha dispuesto de los suficientes instrumentos $y$ ha sido la Administración Regional la que verdaderamente ha hecho el mayor esfuerzo en este campo, coincidiendo con el desarrollo de esta competencia en las Comunidades Autónomas.

\subsection{El turismo rural como desarrollo sostenible}

Estamos ante una actividad que está claramente alineada en el desarrollo sostenible, en ese principio $2^{\circ}$ de la Declaración sobre el Medioambiente y el Desarrollo de Río de Janeiro del año 92 que dice que: "el derecho al desarrollo debe ejercerse en forma tal que responda equitativamente a las necesidades de desarrollo y ambientales de las generaciones presentes y futuras".

De todos los turismos, este es el que mejor se inserta dentro del desarrollo sostenible y, para Europa, el Comité de Desarrollo Espacial en los "Principios para una política de ordenación del territorio europeo" en Leipzig, año 1994, señalaba de una manera textual: "A raiz de los drásticos cambios que afectan al sector agrario es preciso orientarse hacia nuevas actividades económicas compatibles con la protección del medio ambiente, ecoturismo, gestión del paisaje, agricultura ecológica, etc. que permitan detener la despoblación del campo, manteniendo sobre el terreno a la población requerida para desarrollar modelos de asentamiento y preservar los paisajes naturales".

La política territorial diseñada en Leipzig para el espacio europeo se asienta geométricamente en la promoción de tres elementos: puntos, ejes y superficies, que deben conectarse entre sí.

Los puntos motrices corresponden al sistema de ciudades generadoras de desarrollo desde una economía servoindustrial. Las infraestructuras e infoestructuras encauzan los intercambios entre las ciudades, tanto de personas como de mercancías, servicios e ideas. Las superficies corresponden a dos tipos: las productivas y las de protección. Éstas últimas no acaban de ser bien encauzadas en la política territorial europea. Los expertos señalan como problema principal la falta de conexión de estos espacios entre sí y con el sistema general. El tratamiento de protecciòn a base de islotes en los ecosistemas empobrece la biodiversidad. 
Por otro lado, hay falta de convergencia de las políticas de protección de patrimonio natural y cultural. Por último, la protección no debe estar reñida con una posible productividad de estos espacios en la medida en que sus atractivos paisajísticos y culturales pueden generar riqueza a través del turismo cultural.

\section{Los parques culturales}

Una respuesta que tiende a resolver los problemas enunciados es el parque cultural, figura creada en Aragón hace más de una década con base en las pinturas rupestres y potenciada en el último cuatrienio con otros elementos del patrimonio cultural y su entorno paisajístico.

La Administración puede no sólo plantear ayudas a la vivienda de turismo rural y promocionarla publicitariamente, sino además procurar atractivos para "vender" el entorno, al mismo tiempo que lo protege.

La ley aragonesa del 5 de diciembre de 1997, aprobada por unanimidad en las Cortes Aragonesas", señala en su preámbulo que los parques culturales "ban demostrado ser un medio eficaz para el desarrollo sostenible en el ámbito rural aragonés".

En el artículo $1^{\circ}$ de la ley se define el parque cultural como "un territorio que contiene elementos relevantes del patrimonio cultural, integrados en un marco físico de valor paisajístico y/o ecológico singular, que gozará de promoción y protección global en su conjunto, con especiales medidas de protección para dichos elementos relevantes".

La ley de parques culturales de Aragón es una normativa de ordenación del territorio articulada por el patrimonio cultural-natural en la búsqueda de un desarollo rural sostenible a partir del turismo. Así, en el artículo $2^{\circ}-3$, se dice que "En el parque cultural deberán coordinarse las politicas territoriales con las sectoriales, especialmente desde patrimonio cultural y natural, fomento de la actividad económica, turismo rural, infraestructuras y equipamientos". Entre los objetivos de los parques, el artículo $3^{\text {o }}$ señala: la protección y conservación del patrimonio, la promoción turística, la ordenación del territorio y el desarrollo rural sostenible "mejorando el nivel y la calidad de vida de las áreas afectadas, con especial atención a los usos y aprovechamientos tradicionales".

La ampliación de la protección del arte rupestre a todas las manifestaciones relevantes del patrimonio cultural hizo que desde la propia ley se pudieran contemplar como parques culturales no sólo los iniciales de Albarracín, Río Vero y Río Martín, sino también el Maestrazgo, en el que el paisaje natural y antrópico integra ricos conjuntos paleontológicos y bienes culturales de singular valor, así

*. El autor de este artículo presentó el proyecto siendo Consejero de Educación y Cultura del Gobierno de Aragón. 
como San Juan de la Peña, cuyos monumentales monasterios se enmarcan en un bellísimo paisaje prepirenaico, salpicado de ermitas.

En un parque cultural, tal y como lo contemplaba la ley aragonesa, se trata de proteger y promocionar al mismo tiempo el patrimonio "ecocultural". La mejor protección, según hemos podido comprobar en Aragón, es implicar a sus habitantes en la puesta en valor de lo que es hoy un patrimonio económico, más valioso que los recursos agrarios. La identificación, conocimiento y valorización de los bienes de interés cultural y ecológico por parte de los campesinos, en orden a su "venta turística", significa acabar con los expolios, mejorar el cuidado del paisaje y aumentar los ingresos de unas poblaciones de áreas hasta ahora deprimidas y con tendencia a la despoblación, como son las de la montaña media aragonesa.

\section{Agradecimientos}

Este artículo se enmarca en el proyecto de investigación "Desarrollo sostenible y medio ambiente en las áreas de montaña. El turismo rural" financiado por la DGICYT y dirigido inicialmente por el autor y con posterioridad por $\mathrm{D}^{\mathrm{a}}$ Blanca Loscertales.

\section{Bibliografía}

Alonso PÉrez, M. (1991): El agroturismo: una alternativa para las áreas rurales valencianas. Noticias de la Economía Pública Social y Cooperativa, núm. 9: 2126 págs.

BARTHEZ, A. (1984): Femmes dans l'agriculture et travail familial. Sociologie du Travail, núm. 3: 46-72 págs.

Bielza, V. y de Miguel, R. (1997): El patrimonio cultural, componente de la ordenación del territorio. Revista Aragonesa de Administración Pública, núm. 10. Gobierno de Aragón.

Bryan, B. (1991): Ecoturism on Family Farms and Ranches in the American West. En T. Whelan (Ed.): In Tourism: Managing for the Environment. Whashington. Island Press, 75-85 págs.

Canoves, G. (1995): Estructura familiar i treball de la dona a l'agricultura: E1 cas d'Osona i El Baix Empordá. Documents d'Analisi Geográfica, núm. 26: 53-71.

Cruz, J. (1991): Nueva dinámica de los espacios rurales, en C. Domingo (Ed.): Asociación de geógrafos españoles, XII Congreso de Geografia, Sociedad y Territorio. Valencia. Universidad de Valencia, 257-267 págs.

CUTTER, S.L. (1994): Environmental issues: Green rage, social change and the new environmentalism. Progress in Human Geograpby, 18 (2), 217-226 págs.

El Campo (1994): Número especial dedicado a Agricultura y Medio Ambiente, 131, 5-249 págs. 
El CAMPo (1995): Número especial dedicado a la mujer rural, 133, 11-313 págs.

ETXEZARRETA, M. (1994): De la agricultura familiar al desarrollo rural. En VV.AA. Medio Ambiente y Ordenación del Territorio, Universidad de Valladolid, Valladolid.

García RAMÓn, M.D. (1992): Desarrollo y tendencias actuales de la Geografía Rural (1980-1990). Una perspectiva internacional y una agenda para el futuro, VI Coloquio de Geografía Rural. Ponencias, Departamento de Geografía Universidad Autónoma de Madrid, Madrid.

Gómez GIL, J.L. (1992): Medidas comunitarias para el fomento del turismo rural, en S. Reyna (Ed.): El turismo rural en el desarrollo local. Madrid. Ministerio de Agricultura. 39-50 págs.

KOWALCZYK, A. (1994): The role of tourism in local development. Comunicación presentada en la sesión E.3.5 sobre "Recreation and Tourism", International Geographical Unión Regional Conference, Praga, Agosto (mimeo).

Lowe P. y otros (Eds) (1993): Cambio Tecnológico y Medio Ambiente Rural, Ministerio de Agricultura, Secretaría General Técnica, Madrid.

Whatmore, S. (1993): Sustainable rural policies?, Progress in Human Geography, 17 (4), 538-547 págs.

Wimberley, R. (1993): Policy perspectives on Social, Agricultural, and Rural Sustainability, Rural Sociology, 58 (1), 1 págs. 